\title{
Understanding the Dynamic Potential Distribution at the Electrode Interface by Stochastic Collision Electrochemistry
}

Si-Min Lu ${ }^{1,3 \ddagger}$, Jian-Fu Chen ${ }^{2 \ddagger}$, Yue-Yi Peng ${ }^{1,3 \ddagger}$, Wei Ma ${ }^{3}$, Hui Ma ${ }^{1,3}$, Hai-Feng Wang ${ }^{2}$, Peijun $\mathrm{Hu}^{2,4}$, Yi-Tao Long ${ }^{1,3 *}$

${ }^{1}$ State Key Laboratory of Analytical Chemistry for Life Science, School of Chemistry and Chemical Engineering, Nanjing University, Nanjing 210023, P. R. China

${ }^{2}$ State Key Laboratory of Chemical Engineering, Centre for Computational Chemistry \& Research Institute of Industrial Catalysis, East China University of Science and Technology, Shanghai 200237, P. R. China

${ }^{3}$ School of Chemistry and Molecular Engineering, East China University of Science and Technology, Shanghai 200237, P. R. China

${ }^{4}$ School of Chemistry and Chemical Engineering, The Queen's University of Belfast, Belfast, BT9 5AG, UK

\$These authors contributed equally: Si-Min Lu, Jian-Fu Chen, Yue-Yi Peng

*Correspondence should be addressed to Y.-T. L. (yitaolong@ @ju.edu.cn)

\section{Table of Contents}

1. Supplementary Notes 1

Supplementary Note 1 Simmons model for tunneling in M-S-MNP system----------------2

Supplementary Note 2 Geometric correction in M-S-MNP system----------------------- 4

Supplementary Note 3 Electrochemical kinetics in M-S-MNP system ---------------------7

Supplementary Note 4 Materials and methodology -------------------------------------9

2. Supplementary Figures

Supplementary Figure S1 Differential-pulse voltammetry of Ag electro-oxidation-----11

Supplementary Figure S2 Effective potential curve and tunneling barrier energy------ 12

Supplementary Figure S3 Details of simulation for M-S-MNP system ---------------15

Supplementary Figure S4 Simulated 2D contour map in M-S-MNP system------------17

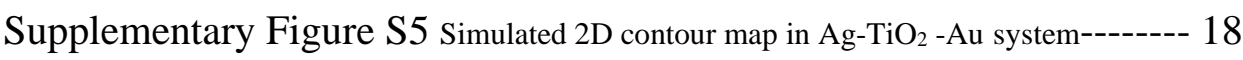

Supplementary Figure S6 Statistic results electrooxidation of single Ag NPs-----------19 


\section{Supplementary Notes}

\section{Supplementary Note 1: Simmons model for tunneling in the M-S-MNP system}

The Simmons theory is used to describe the tunneling current between two electrodes, which are separated by a thin insulating film. Here, considering the fact that the low energy barrier of electrolyte solution between Au UME and Ag NPs, we innovatively adapt Simmons model (with only one little approximations) into single Ag NPs collision system by treating Au UME and individual Ag NPs as two dissimilar electrodes in the electrolyte solution.

Following Simmons, the form for the tunneling current from metal 1 to metal 2 in a planar MIM structure $^{1,2}$ can be written as follows after considerable deduction: ${ }^{3}$

$J=\frac{4 \pi q m}{\mathrm{~h}^{3}} \iint[f(E)-f(E+\Delta E)] P_{t}\left(E_{x}\right) d E_{x} d E_{t}$

where the electrochemical potential energy difference between two metals is $\Delta E=\Delta \psi+q \eta$, $q$ is the elementary charge, $\eta$ is the applied overpotential, the effect of dissimilar metal is to produce an asymmetric potential barrier, the barrier heights differing by $\Delta \psi=\psi_{2}-\psi_{1}, \psi_{1}$ is the work function of $\mathrm{Ag} \mathrm{NP}, \psi_{2}$ is the work function of Au UME, $m$ is the electronic mass, $\mathrm{h}$ is the Planck constant, $E_{x}$ and $E_{t}$ are the energy of electron $(E)$ by decomposing into parallel $\left(E_{x}\right)$ and "transverse" $\left(E_{t}\right)$ components, $f(E)$ represent the probability being occupied in metal 1/2 according to Fermi-Dirac statistics:

$f(E)=\frac{1}{1+e^{\frac{E-E_{F}}{\mathrm{k}_{\mathrm{b}} T}}}$

where $E_{F}$ is the energy of Fermi level, $k_{\mathrm{b}}$ is Boltzmann constant, $T$ is the temperature. Due to the fact that for a positive overpotential, $f(E)-f(E+\Delta E)$ will be 1 when $\left(E_{F}-\Delta E\right)<$ $E \leq E_{F}$ and 0 otherwise, equation 1 can then be simplified to: ${ }^{3}$

$J=\frac{4 \pi q m}{h^{3}}\left[\Delta E \int_{0}^{E_{F}-\Delta E} P_{t} d E_{x}+\int_{E_{F}-\Delta E}^{E_{F}}\left(E_{F}-E_{\chi}\right) P_{t} d E_{\chi}\right]$

and $P_{t}$ is the probability of tunneling through the barrier, which is taken to be:

$P_{t}=e^{-A\left(\bar{E}_{b}-E_{x}\right)^{1 / 2}}$

where $\bar{E}_{b}$ is the energy level of the tunneling barrier, $A=2 w \sqrt{\frac{2 m}{\hbar^{2}}}=4 \sqrt{2 m} \pi w / h ; w$ is the thickness of the tunneling barrier, $\hbar$ is the reduced Planck constant $(\mathrm{h} /(2 \pi)) . h$ is the Planck constant, $J$ then becomes:

$J=\frac{4 \pi q m}{h^{3}}\left[I_{1}+I_{2}+I_{3}\right]$ 


$$
\begin{aligned}
& I_{1}=\Delta E \int_{0}^{E_{F}-\Delta E} e^{-A\left(\bar{E}_{b}-E_{\chi}\right)^{1 / 2}} d E_{x} \\
& I_{2}=E_{F} \int_{E_{F}-\Delta E}^{E_{F}} e^{-A\left(\bar{E}_{b}-E_{\chi}\right)^{1 / 2}} d E_{x} \\
& I_{3}=-\int_{E_{F}-\Delta E}^{E_{F}} E_{x} e^{-A\left(\bar{E}_{b}-E_{\chi}\right)^{1 / 2}} d E_{x}
\end{aligned}
$$

Each of these integrals can be evaluated in a straightforward manner through the integration by parts to yield:

$$
\begin{aligned}
& I_{1}=\frac{2 \Delta E}{A^{2}}\left\{\left[1+A\left(\bar{E}_{b}-E_{F}+\Delta E\right)^{1 / 2}\right] e^{-A\left(\bar{E}_{b}-E_{F}+\Delta E\right)^{1 / 2}}-\left(1+A \bar{E}_{b}^{1 / 2}\right) e^{-A \bar{E}_{b}^{1 / 2}}\right\} \\
& I_{2}=\frac{2 E_{F}}{A^{2}}\left\{\left[1+A\left(\bar{E}_{b}-E_{F}\right)^{\frac{1}{2}}\right] e^{-A\left(\bar{E}_{b}-E_{F}\right)^{\frac{1}{2}}}-\left[1+A\left(\bar{E}_{b}-E_{F}+\Delta E\right)^{\frac{1}{2}}\right] e^{-A\left(\bar{E}_{b}-E_{F}+\Delta E\right)^{\frac{1}{2}}}\right\}(6) \\
& I_{3}=\left.\left\{\frac{2 e^{-A\left(\bar{E}_{b}-E_{x}\right)^{1 / 2}}}{A^{4}}\left[A^{3} E_{x}\left(\bar{E}_{b}-E_{x}\right)^{1 / 2}+A^{2}\left(3 E_{x}-2 \bar{E}_{b}\right)-6 A\left(\bar{E}_{b}-E_{x}\right)^{1 / 2}-6\right]\right\}\right|_{E_{F}} ^{E_{F}-\Delta E}
\end{aligned}
$$

By only neglecting the $e^{-A \bar{E}_{b}^{1 / 2}}$ terms, $J$ can then be evaluated to yield:

$J=\frac{16 \pi q m}{A^{4} h^{3}}\left\{e^{-A \sqrt{\bar{\varphi}}}\left[A^{2} \bar{\varphi}+3 A \sqrt{\bar{\varphi}}+3\right]-e^{-A \sqrt{\bar{\varphi}+\Delta E}}\left[A^{2}(\bar{\varphi}+\Delta E)+3 A \sqrt{\bar{\varphi}+\Delta E}+3\right]\right\}(7)$ where $\bar{\varphi}=\bar{E}_{b}-E_{F}$ is the tunneling barrier.

For the typical parameter values of two electrodes which are separated by a thin insulating film (i.e. high tunneling barrier $\bar{\varphi}=1 \mathrm{eV}$ and $\mathrm{A}=2 a \mathrm{w}=2 \mathrm{w} \sqrt{\frac{2 m}{\hbar^{2}}}, a=\sqrt{\frac{2 m}{\hbar^{2}}} \approx$ $\left.0.512 \mathrm{eV}^{1 / 2} \AA^{-1}\right)$, by only keep the first term $\left(A^{2} \bar{\varphi}\right.$ and $\left.A^{2}(\bar{\varphi}+\Delta E)\right)$, one arrives at the famous result of Simmons model: $J \approx \frac{16 \pi q m}{A^{2} h^{3}}\left[\bar{\varphi} e^{-A \sqrt{\bar{\varphi}}}-(\bar{\varphi}+\Delta E) e^{-A \sqrt{\bar{\varphi}+\Delta E}}\right]$.

If we defined the function $F_{J}(A, x)=\frac{16 \pi q m}{A^{4} h^{3}} e^{-A \sqrt{x}}\left(A^{2} x+3 A \sqrt{x}+3\right)$, the forward and backward tunneling currents are $J_{f}=F_{J}(A, \bar{\varphi})$ and $J_{b}=F_{J}(A, \bar{\varphi}+\Delta E)$, respectively, and substituted $J=J_{f}-J_{b}=F_{J}(A, \bar{\varphi})-F_{J}(A, \bar{\varphi}+\Delta E)$. 


\section{Supplementary Note 2: Geometric correction in the M-S-MNP system}

The tunneling current in the MSM system is then:

$i_{\text {tun }}=\int_{w_{0}}^{w_{0}+r_{0}} J \cdot d S=\int_{0}^{\frac{\pi}{2}} J \cdot 2 \pi r_{0} \sin \theta r_{0} d \theta$

where the tunneling distance $w(\theta)=w_{0}+r_{0}-r_{0} \cos \theta$, then $d w=-r_{0} d \cos \theta=$ $r_{0} \sin \theta d \theta$.

$$
i_{\text {tun }}=\int_{0}^{\frac{\pi}{2}} J \cdot 2 \pi r_{0} \sin \theta r_{0} d \theta=2 \pi r_{0} \int_{w_{0}}^{w_{0}+r_{0}} J \cdot d w=\frac{\pi r_{0}}{a} \int_{2 a w_{0}}^{2 a\left(w_{0}+r_{0}\right)} J \cdot d A
$$

If we defined the function $i(w, x)=\frac{\pi r_{0}}{a} \int_{2 a w_{0}}^{2 a\left(w_{0}+r_{0}\right)} F_{J}(A, x) \cdot d A$ and substituted $J=J_{f}-J_{b}$ $=F_{J}(A, \bar{\varphi})-F_{J}(A, \bar{\varphi}+\Delta E), i_{\text {tun }}$ can then be simplified to yield:

$$
i_{\text {tun }}=\frac{\pi r_{0}}{a} \int_{2 a w_{0}}^{2 a\left(w_{0}+r_{0}\right)} J_{f} \cdot d A-\frac{\pi r_{0}}{a} \int_{2 a w_{0}}^{2 a\left(w_{0}+r_{0}\right)} J_{b} \cdot d A=i(w, \bar{\varphi})-i(w, \bar{\varphi}+\Delta E)
$$

Each of these integrals can be evaluated through integration by parts as following forms:

$\int \frac{e^{-c y}}{y^{n}} d y=-\frac{1}{n-1} \frac{e^{-c y}}{y^{n-1}}-\int \frac{c}{n-1} \frac{e^{-c y}}{y^{n-1}} d y$

To make an easy transformation of $i_{\text {tun }}, G_{i}(w, x)$ was defined in an improper integral manner as following forms:

$$
\begin{aligned}
& G_{i}(w, x) \\
& =\frac{\pi r_{0}}{a} \int F_{J}(A, x) d A \\
& =\frac{\pi r_{0}}{a} \int \frac{16 \pi q m}{A^{4} h^{3}} e^{-A \sqrt{x}}\left(A^{2} x+3 A \sqrt{x}+3\right) d A \\
& =\frac{16 \pi^{2} q m r_{0}}{a h^{3}} \int\left(\frac{3 e^{-A \sqrt{x}}}{A^{4}}+\frac{3 \sqrt{x} e^{-A \sqrt{x}}}{A^{3}}+\frac{x e^{-A \sqrt{x}}}{A^{2}}\right) d A=-\frac{16 \pi^{2} q m r_{0}}{a A^{3} h^{3}} e^{-A \sqrt{x}}(A \sqrt{x}+1) \\
& =-\frac{q h r_{0}}{32 \pi^{2} m w^{3}} e^{-2 a w \sqrt{x}}(2 a w \sqrt{x}+1)
\end{aligned}
$$

Thus, $i(w, x)$ can be further written as:

$i(w, x)=\frac{\pi r_{0}}{a} \int_{2 a w_{0}}^{2 a\left(w_{0}+r_{0}\right)} F_{J}(A, x) d A=\left.G_{i}(A, x)\right|_{2 a w_{0}} ^{2 a\left(w_{0}+r_{0}\right)}=G_{i}\left(w_{0}+r_{0}, x\right)-G_{i}\left(w_{0}, x\right)($

And the $i_{\text {tun }}$ is given by: 


$$
\begin{aligned}
& i_{\text {tun }}=\frac{\pi r_{0}}{a} \int_{2 a w_{0}}^{2 a\left(w_{0}+r_{0}\right)} J \cdot d A=i(w, \bar{\varphi})-i(w, \bar{\varphi}+\Delta E) \\
& =\left[G_{i}\left(w_{0}+r_{0}, \bar{\varphi}\right)-G_{i}\left(w_{0}, \bar{\varphi}\right)\right]-\left[G_{i}\left(w_{0}+r_{0}, \bar{\varphi}+\Delta E\right)-G_{i}\left(w_{0}, \bar{\varphi}+\Delta E\right)\right] \\
& =\frac{q h r_{0}}{32 \pi^{2} m w_{0}^{3}}\left[e^{-2 a w_{0} \sqrt{\bar{\varphi}}}\left(2 a w_{0} \sqrt{\bar{\varphi}}+1\right)-e^{-2 a w_{0} \sqrt{\bar{\varphi}+\Delta E}}\left(2 a w_{0} \sqrt{\bar{\varphi}+\Delta E}+1\right)\right] \\
& -\frac{q h r_{0}}{32 \pi^{2} m\left(w_{0}+r_{0}\right)^{3}}\left[e^{-2 a\left(w_{0}+r_{0}\right) \sqrt{\bar{\varphi}}}\left(2 a\left(w_{0}+r_{0}\right) \sqrt{\bar{\varphi}}+1\right)\right. \\
& \left.-e^{-2 a\left(w_{0}+r_{0}\right) \sqrt{\bar{\varphi}+\Delta E}}\left(2 a\left(w_{0}+r_{0}\right) \sqrt{\bar{\varphi}+\Delta E}+1\right)\right]
\end{aligned}
$$

Although the variation of the tunneling current $i_{\text {tun }}$ with the tunneling distance $\left(w_{0}\right)$, nanoparticle radius $\left(r_{0}\right)$, or the potential difference $(\Delta E)$ etc. can be viewed by the numeric simulation with the above complex equation. The correlations between them are still difficult to understand intuitively due to the subtraction. Thus, the equation is further simplified by some reasonable approximations.

Case 1: when the magnitude of $\bar{\varphi}$ is larger than that of $\Delta E$

If we defined the function $i_{t u n}(w)=-G_{i}(w, \bar{\varphi})+G_{i}(w, \bar{\varphi}+\Delta E)$, thus, the $i_{\text {tun }}$ can be rewritten as $i_{\text {tun }}=i_{\text {tun }}\left(w_{0}\right)-i_{\text {tun }}\left(w_{0}+r_{0}\right)$.

If the electrochemical potential energy difference between two metals $\Delta E$ is very small. For typical parameter values $a \varphi^{1 / 2} w \gg 1$, the follows can be further simplified as:

$$
\begin{aligned}
& i_{\text {tun }}(w)=-G_{i}(w, \bar{\varphi})+G_{i}(w, \bar{\varphi}+\Delta E) \\
& =\frac{q h r_{0}}{32 \pi^{2} m w^{3}}\left[e^{-2 a w \sqrt{\bar{\varphi}}}(2 a w \sqrt{\bar{\varphi}}+1)-e^{-2 a w \sqrt{\bar{\varphi}+\Delta E}}(2 a w \sqrt{\bar{\varphi}+\Delta E}+1)\right] \\
& \approx \frac{q a h r_{0}}{16 \pi^{2} m w^{2}}\left(\sqrt{\bar{\varphi}} e^{-2 a w \sqrt{\bar{\varphi}}}-\sqrt{\bar{\varphi}+\Delta E} e^{-2 a w \sqrt{\bar{\varphi}+\Delta E}}\right)
\end{aligned}
$$

A linear $J-\Delta E$ relation can be found for small overpotentials. This can be done by assuming $\Delta E$ is small and finding $\left(\varphi-\frac{\Delta E}{2}\right)^{1 / 2} \approx \varphi^{\frac{1}{2}}\left(1-\frac{\Delta E}{4 \varphi}\right)$ through series expansion, expanding the exponentials $\left(e^{c y} \approx 1+c y\right)$ and simplifying results in:

$$
i_{\text {tun }}(w) \approx \frac{q \Delta E a^{2} h r_{0}}{16 \pi^{2} m w} e^{-2 a w \sqrt{\varphi}}\left(1-\frac{1}{a w \sqrt{\varphi}}\right)
$$

When $a \varphi^{1 / 2} w \gg 1$, this simplifies to:

$$
i_{t u n}(w) \approx \frac{q \Delta E r_{0}}{2 h w} e^{-2 a w \sqrt{\bar{\varphi}}}
$$

Finally, $i_{\text {tun }}$ is then:

$$
i_{\text {tun }}=i_{\text {tun }}\left(w_{0}\right)-i_{\text {tun }}\left(w_{0}+r_{0}\right)=\frac{q \Delta E r_{0}}{2 h} e^{-2 a w_{0} \sqrt{\bar{\varphi}}}\left(\frac{1}{w_{0}}-\frac{e^{-2 a r_{0} \sqrt{\bar{\varphi}}}}{w_{0}+r_{0}}\right)
$$


The validity of this approximate form for $i_{\text {tun }}$ can be checked. If assuming $\Delta E$ is small, equation 3 can be simplified to be:

$J=\frac{4 \pi q m}{h^{3}}\left[\Delta E \int_{0}^{E_{F}} P_{t} d E_{x}\right]$

By only neglecting the $e^{-A \bar{E}_{b}^{1 / 2}}$ terms, $J$ can then be evaluated to yield:

$J=\frac{8 \pi q \Delta E m}{A^{2} h^{3}} e^{-A \sqrt{\bar{\varphi}}}(A \sqrt{\bar{\varphi}}+1)$

Then correcting by Geometry according to equation 9 ,

$$
\begin{aligned}
& i_{\text {tun }}=\frac{\pi r_{0}}{a} \int_{2 a w_{0}}^{2 a\left(w_{0}+r_{0}\right)} J \cdot d A=\left.\frac{8 \pi q \Delta E m r_{0}}{A a h^{3}} e^{-A \sqrt{\bar{\varphi}}}\right|_{2 a w_{0}} ^{2 a\left(w_{0}+r_{0}\right)}=\left.\frac{q \Delta E r_{0}}{2 h w} e^{-2 a w \sqrt{\bar{\varphi}}}\right|_{w_{0}} ^{w_{0}+r_{0}}= \\
& -\left.i_{\text {tun }}(w)\right|_{w_{0}} ^{w_{0}+r_{0}}
\end{aligned}
$$

Which is consisted with the equation 18.

For the typical parameter value $r_{0}$ is large, then $i_{t u n}=\frac{q \Delta E r_{0}}{2 h w_{0}} e^{-2 a w_{0} \sqrt{\bar{\varphi}}}$

Or, if normalized by the NP area:

$j_{\text {tun }}=\frac{i_{\text {tun }}}{4 \pi r_{0}^{2}}=\frac{q \Delta E}{8 \pi h w_{0} r_{0}} e^{-2 a w_{0} \sqrt{\bar{\varphi}}}=\frac{q \Delta E_{\max }}{8 \pi h w_{0} r_{0}} \frac{\Delta E}{\Delta E_{\max }} e^{-2 a w_{0} \sqrt{\bar{\varphi}}}=j_{\text {tun }, 1} \frac{\Delta E}{\Delta E_{\max }}$

Case 2: when the magnitude of $\bar{\varphi}$ is smaller than that of $\Delta E$

If the electrochemical potential energy difference between two metals $\Delta E$ is very large, the $i(w, \bar{\varphi}+\Delta E)$ term can be neglected, thus the $i_{\text {tun }}$ is then:

$i_{\text {tun }} \approx i(w, \bar{\varphi})=G_{i}\left(w_{0}+r_{0}, \bar{\varphi}\right)-G_{i}\left(w_{0}, \bar{\varphi}\right)$

For the typical parameter value $r_{0}$ is large, then

$i_{\text {tun }} \approx G_{i}\left(w_{0}+r_{0}, \bar{\varphi}\right)-G_{i}\left(w_{0}, \bar{\varphi}\right) \approx-G_{i}\left(w_{0}, \bar{\varphi}\right)=\frac{q h r_{0}}{32 \pi^{2} m w_{0}^{3}}\left[e^{-2 a w_{0} \sqrt{\bar{\varphi}}}\left(2 a w_{0} \sqrt{\bar{\varphi}}+1\right)\right] \approx$

$\frac{q \sqrt{2 \bar{\varphi}} r_{0}}{8 \pi \sqrt{m} w_{0}^{2}} e^{-2 a w_{0} \sqrt{\bar{\varphi}}}$

Or, if normalized by the NP area:

$$
j_{\text {tun }}=\frac{i_{t u n}}{4 \pi r_{0}^{2}}=\frac{q \sqrt{2 \bar{\varphi}}}{32 \pi^{2} \sqrt{m} w_{0}^{2} r_{0}} e^{-2 a w_{0} \sqrt{\bar{\varphi}}}=j_{t u n, 2} e^{-2 a w_{0} \sqrt{\bar{\varphi}}}
$$

Thus, the tunneling current can be summarized as

$$
i_{\text {tun }}=\left\{\begin{array}{c}
\frac{q \Delta E r_{0}}{2 h w_{0}} e^{-2 a w_{0} \sqrt{\bar{\varphi}}}, \text { large } \bar{\varphi} \\
\frac{q \sqrt{2 \bar{\varphi}} r_{0}}{8 \pi \sqrt{m} w_{0}^{2}} e^{-2 a w_{0} \sqrt{\bar{\varphi}}}, \text { otherwise }
\end{array}\right.
$$


Or, if normalized by the NP area: $j_{\text {tun }}=\left\{\begin{array}{c}\frac{q \Delta E}{8 \pi h w_{0} r_{0}} e^{-2 a w_{0} \sqrt{\bar{\varphi}}}, \text { large } \bar{\varphi} \\ \frac{q \sqrt{2 \bar{\varphi}}}{32 \pi^{2} \sqrt{m} w_{0}^{2} r_{0}} e^{-2 a w_{0} \sqrt{\bar{\varphi}}}, \text { otherwise }\end{array}\right.$

\section{Supplementary Note 3: Electrochemical kinetics in the M-S-MNP system}

According to Butler-Volmer model, the Faradaic current of an Ag NP dissolution $\left(i_{o x}\right)$ is:

$i_{o x}=\frac{\partial Q}{\partial t}=-\frac{F \rho}{M_{A g}} \frac{\partial V}{\partial t}=-\frac{4 \pi r_{0}^{2} F \rho}{M_{A g}} \frac{\partial r_{0}}{\partial t}=-\frac{4 \pi r_{0}^{2} F \rho}{M_{A g}} v_{r}\left(e^{\frac{-\alpha q\left(U_{p}-U^{o}\right)}{k_{b} T}}-e^{\frac{(1-\alpha) q\left(U_{p}-U^{o}\right)}{k_{b} T}}\right)$

where $F$ is Faraday's constant, $\rho$ is the density of $\mathrm{Ag}, M_{\mathrm{Ag}}$ is the atomic molecular mass of $\mathrm{Ag}$ and $v_{r}$ is the electrochemical reaction rate at the equilibrium potential, $\eta_{p}=U_{p}-U^{o}$ is the overpotential for electro-oxidation of single Ag NPs, and $U^{o}$ is the equilibrium potential for the Ag NPs electro-oxidation. In steady-state condition, the oxidation current is equal to the tunneling current $\left(i_{o x}=i_{t u n}\right)$. It can be seen that as long as there is a tunneling current, the oxidation current will not be equal to 0 . That means the reaction will not reach equilibrium. Only when the electron tunneling does not occur where the nanoparticle in the bulk solution is far from the electrode surface $\left(U_{p}=U_{\text {bulk }}\right.$ ), both the tunneling current and the oxidation current can be equal to $0\left(i_{o x}=i_{t u n}=0\right)$. At that time, the oxidation reaction can reach equilibrium, which is corresponding to $U_{p}=U_{0}$. Together with the condition $U_{p}=U_{\text {bulk }}$, thus, $U_{0}=U_{\text {bulk }}$, and $U_{\text {bulk }}$ is set as reference for further theoretical simulation as we neglect the trivial solution, then $i_{o x}$ can be simplified to be:

$i_{o x}=-\frac{4 \pi r_{0}^{2} F \rho}{M_{A g}} v_{r}\left(e^{-\frac{\alpha q \eta_{p}}{k_{b} T}}-e^{\frac{(1-\alpha) q \eta_{p}}{k_{b} T}}\right)=\left\{\begin{array}{c}i_{o x, 0} e^{\frac{(1-\alpha) q \eta_{p}}{k_{b} T}}, \text { large } \eta_{p} \\ i_{o x, 0} \frac{q \eta_{p}}{k_{b} T}, \text { otherwise }\end{array}\right.$

where large $\eta_{p}$ corresponds to the condition that $\eta_{p}$ is on the same order of magnitude as $U_{e l}$. Or, if normalized by the NP area:

$j_{o x}=\left\{\begin{array}{c}\frac{F \rho v_{r}}{M_{A g}} e^{\frac{(1-\alpha) q \eta_{p}}{k_{b} T}}=j_{o x, 0} e^{\frac{(1-\alpha) q \eta_{p}}{k_{b} T}}, \text { large } \eta_{p} \\ \frac{q \eta_{p} F \rho v_{r}}{M_{A g} k_{b} T}=j_{o x, 0} \frac{q \eta_{p}}{k_{b} T}, \text { otherwise }\end{array}\right.$

At the steady state, the tunneling current density is equivalent to the oxidation current density, $j_{t u n}=j_{o x}$, then the overpotential for electro-oxidation of single Ag NPs $\eta_{p}$ can be achieved: $\eta_{p}=U_{p}-U^{o}=U_{e l}-U_{b u l k}=\left\{\begin{array}{c}\frac{k_{b} T}{(1-\alpha) q} \ln \frac{j_{t u n}}{j_{o x, 0}}, \text { large } \eta_{p} \\ \frac{k_{b} T j_{t u n}}{q j_{o x, 0}}, \text { otherwise }\end{array}\right.$

then the particle potential energy $U_{p}$ can be achieved: 
$U_{p}=U_{b u l k}+\left\{\begin{array}{c}\frac{k_{b} T}{(1-\alpha) q} \ln \frac{j_{t u n}}{j_{o x, 0}}, \text { large } \eta_{p} \\ \frac{k_{b} T j_{t u n}}{q j_{o x, 0}}, \text { otherwise }\end{array}\right.$

Here, the particle potential $U_{p}$ could be defined relative to the bulk potential $U_{b u l k}$, and $U_{\text {bulk }}$ is set as the reference for the further simulation, thus $U_{p}=\eta_{p}$.

When the magnitude of $\bar{\varphi}$ is larger than that of $\Delta E$,

$U_{p}=U_{b u l k}+\left\{\begin{array}{c}\frac{k_{b} T}{(1-\alpha) q}\left(\ln \frac{q^{2} U_{e l} M_{A g}}{8 \pi h F \rho v_{r} w_{0} r_{0}}-2 a w_{0} \sqrt{\bar{\varphi}}\right), \text { large } U_{p} \\ \frac{q U_{e l} M_{A g} k_{b} T}{8 \pi h F \rho v_{r} w_{0} r_{0}} e^{-2 a w_{0} \sqrt{\bar{\varphi}}}, \text { otherwise }\end{array}\right.$

When the magnitude of $\bar{\varphi}$ is smaller than that of $\Delta E$,

$U_{p}=U_{b u l k}+\left\{\begin{array}{c}\frac{k_{b} T}{(1-\alpha) q}\left(\ln \frac{q \sqrt{2 \bar{\varphi}} M_{A g}}{32 \pi^{2} \sqrt{m} F \rho v_{r} w_{0}{ }^{2} r_{0}}-2 a w_{0} \sqrt{\bar{\varphi}}\right), \text { large } U_{p} \\ \frac{\sqrt{2 \bar{\varphi}} M_{A g} k_{b} T}{32 \pi^{2} \sqrt{m} F \rho v_{r} w_{0}{ }^{2} r_{0}} e^{-2 a w_{0} \sqrt{\bar{\varphi}}}, \text { otherwise }\end{array}\right.$

Only if the $\Delta E$ is very small $\left(U_{p}\right.$ is very close to $\left.U_{e l}\right)$

$U_{p}=U_{e l}\left(1-\frac{j_{o x}}{j_{t u n, 1}}\right)=U_{e l}-\frac{8 \pi h F \rho w_{0} r_{0}}{M_{A g} q^{2}} v_{r} e^{\frac{(1-\alpha) q\left(U_{e l}-U_{b u l k}\right)}{k_{b} T}+2 a w_{0} \sqrt{\bar{\varphi}}}$

This means that the particle potential $U_{p}$ will vary from the potential of the bulk solution $U_{b u l k}$ to electrode potential $U_{e l}$. 


\section{Supplementary Note 4: Materials and Methodology}

Chemicals and materials. Silver nanoparticles (Ag NPs) capped with citrate ions with the average nominal radius of $10 \mathrm{~nm}$ were purchased from nanoComposix, Inc. (San Diego, CA, USA). The Ag NPs were then capped by carboxyl-terminated alkanethiols through ligand replacement, which was accomplished by mixing the as-prepared Ag NPs with self-assembled monolayers (SAMs) of different lengths (C2 (2-mercaptoacetic acid) C3 (3-mercaptopropanoic acid), C6 (6-mercaptohexanoic acid), C11 (11-mercaptoundecanoic acid), or C16 (16mercaptohexadecanoic acid)) at Ag NPs to alkane thiol molar ratios, respectively (all chemicals are from Sigma Aldrich). After the overnight replacement reaction, the solution was centrifuged and then filtered through a $0.2 \mu \mathrm{m}$ filter to remove aggregates above $200 \mathrm{~nm}$. Other analytical grade reagents were purchased from Sigma-Aldrich. $\mathrm{N}_{2}(99.998 \%$, purified) was obtained from Cryogenic Gases (Detroit, MI). Millipore water (Milli-Q system) purified to a resistivity of 18.2 $\mathrm{M} \Omega \bullet \mathrm{cm}$ with a UHQ II system (Elga) was used to prepare all the solutions. $20 \mathrm{mM}$ Phosphate buffer solution ( $\mathrm{PB}, \mathrm{pH}=7.4)$ was prepared using $\mathrm{Na}_{2} \mathrm{HPO}_{4}$ and $\mathrm{NaH}_{2} \mathrm{PO}_{4}$. The electrolyte solutions were prepared with Millipore water and de-aerated by purging with $\mathrm{N}_{2}$ for $20 \mathrm{~min}$ before experiments, and an atmosphere of $\mathrm{N}_{2}$ was maintained during the experiments.

Table S1. Detailed molecular formula of SAMs

\begin{tabular}{|c|c|c|c|c|c|}
\hline $\begin{array}{l}\text { Molecular } \\
\text { Formula }\end{array}$ & $\mathrm{CH}_{2} \mathrm{SHCOOH}$ & $\mathrm{CH}_{2} \mathrm{SHCH}_{2} \mathrm{COOH}$ & $\mathrm{CH}_{2} \mathrm{SH}\left(\mathrm{CH}_{2}\right)_{4} \mathrm{COOH}$ & $\mathrm{CH}_{2} \mathrm{SH}\left(\mathrm{CH}_{2}\right)_{9} \mathrm{COOH}$ & $\mathrm{CH}_{2} \mathrm{SH}\left(\mathrm{CH}_{2}\right)_{14} \mathrm{COOH}$ \\
\hline Abbreviation & $\mathrm{C} 2$ & $\mathrm{C} 3$ & C6 & $\mathrm{C} 11$ & $\mathrm{C} 16$ \\
\hline $\begin{array}{c}\text { Structural } \\
\text { Formula }\end{array}$ & & & & & \\
\hline Chain Length & $1.5 \AA$ & $2.5 \AA$ & $5.4 \AA$ & $12.7 \AA$ & $18.2 \AA$ \\
\hline
\end{tabular}

Electrochemical measurements. Chronoamperometric curves were obtained using Axon 200B with the two-electrode cell system (a 12,5 $\mu \mathrm{m}$ diameter Au ultramicroelectrode and a $\mathrm{Ag} / \mathrm{AgCl} \mathrm{QRCE}$ electrode). The low-pass Bessel filter was set to $5 \mathrm{kHz}$. The sampling rate controlled by the DigiData 1440A converter and a PC running PClamp 10.4 (Axon Instrument, Forest City, CA) was set to $100 \mathrm{kHz}$. All electrodes were purchased from Shanghai Chenhua Co., Ltd., China. In the electrochemical studies. All stochastic collision electrochemistry of Ag NPs were performed in a double Faraday cage at $25^{\circ} \mathrm{C}$. The raw data was analyzed by using our self-developed software. Differential-pulse voltammetry was used for electrochemical measurements with the Ag NPs-modified $\mathrm{Au}$ electrode as the working electrode, $\mathrm{Ag} / \mathrm{AgCl}$ as 
the quasi-reference counter electrode while a Pt wire electrode acted as a counter electrode (at $25^{\circ} \mathrm{C}$ ), with the $20 \mathrm{mM}$ PB buffer solutions degassed with $\mathrm{N}_{2}$ prior to use. All three electrode electrochemical experiments were performed using a CHI660E electrochemical workstation.

DFT calculation. A Perdew-Burke-Ernzerhof functional within the generalized gradient approximation is used for all the spin-polarized calculations via the VASP code. ${ }^{3-5}$ The optB88 functional with the self-consistent vdW interaction is included. ${ }^{6,7}$ To represent the core-valence electron interactions, the project-augmented wave method was used here. ${ }^{8,9}$ To model the $\mathrm{Au}$ UME and Ag NPs, a four-layer $p(3 \times 3) \mathrm{Au}(111)$ and $\mathrm{Ag}(111)$ surface slab corresponding to 36 $\mathrm{Au}$ atoms and $36 \mathrm{Ag}$ atoms were constructed with a $5 \times 5 \times 1 k$-point sampling, respectively. Also, to further avoid interactions between periodically repeated slabs, a vacuum layer of $25 \AA$ was applied along the z-direction, $\mathrm{Au} 5 d^{10} 6 s^{1}$ and $\mathrm{Ag} 4 d^{10} 5 s^{1}$ electrons were treated as valence electrons. For total energy calculations, the force convergence criterion in the structure was assumed as $0.05 \mathrm{eV} \AA^{-1}$. Additionally, the valence electronic states were expanded in plane wave basis sets with an energy cutoff of $500 \mathrm{eV}$. The bottom two layers of the substrates were fixed, and other atoms were relaxed in the process of structural optimization. The solvent effects were coupled with the polarizable continuum model to simulate the interface solution environment. ${ }^{10,11}$ To further estimate the interactions between Au UME and Ag NPs, the fourlayer $\operatorname{Ag}(111)$ surfaces of $\operatorname{Ag}$ particle with $p(3 \times 3)$ were first constructed to $\operatorname{Au}(111)$ surface as much as possible (2.2\% lattice mismatch). The vacuum and water layers are then added and constrained the different distances between $\mathrm{Ag}(111)$ and $\mathrm{Au}(111)$ surface. Here, the effects of capping agent, charge, and electrolyte on theoretical energy calculation were ignored. ${ }^{12}$ To obtain the information of chain length, DFT calculations were preformed to identify the optimal configures of SAMs at the PM6 level by Gaussian 09. 


\section{Supplementary Figures}

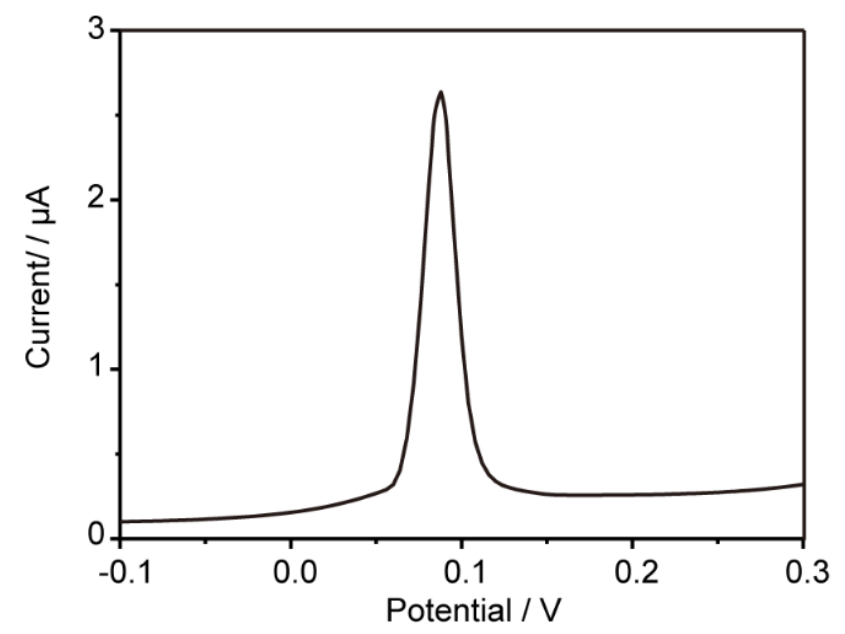

Figure S1. Differential-pulse voltammetry of $20 \mathrm{~nm}$ Ag NPs-modified Au electrode (diameter $2 \mathrm{~mm})$ at a scan rate of $50 \mathrm{mV} \mathrm{s}^{-1}$. Solution contains $20 \mathrm{mM} \mathrm{PB}(\mathrm{pH}=7.4)$. 
a

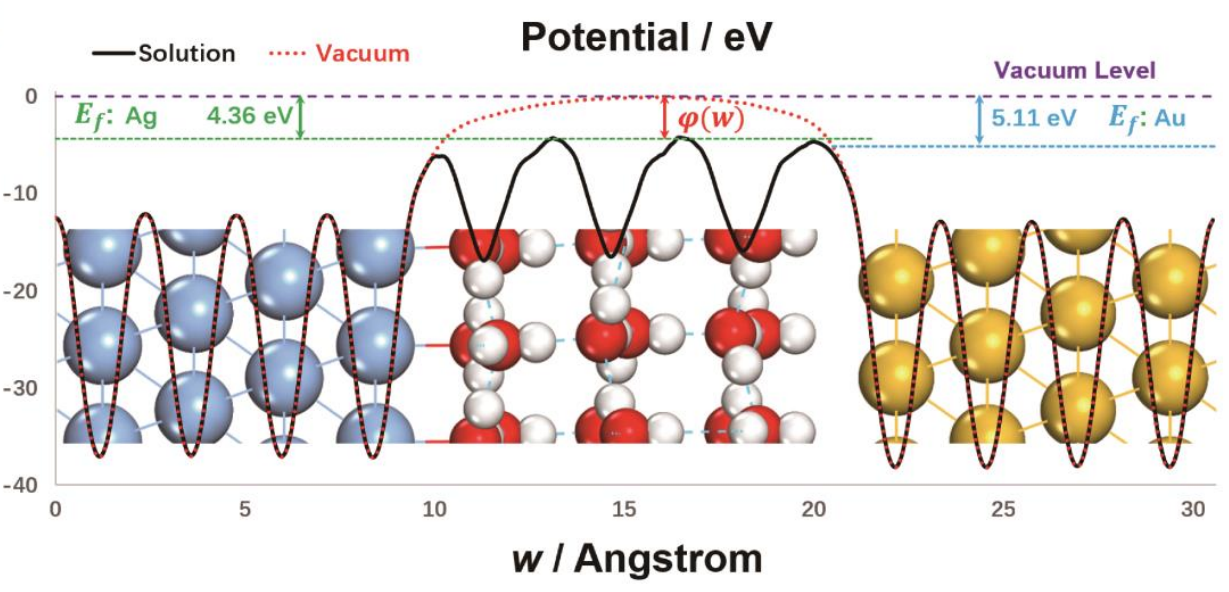

b

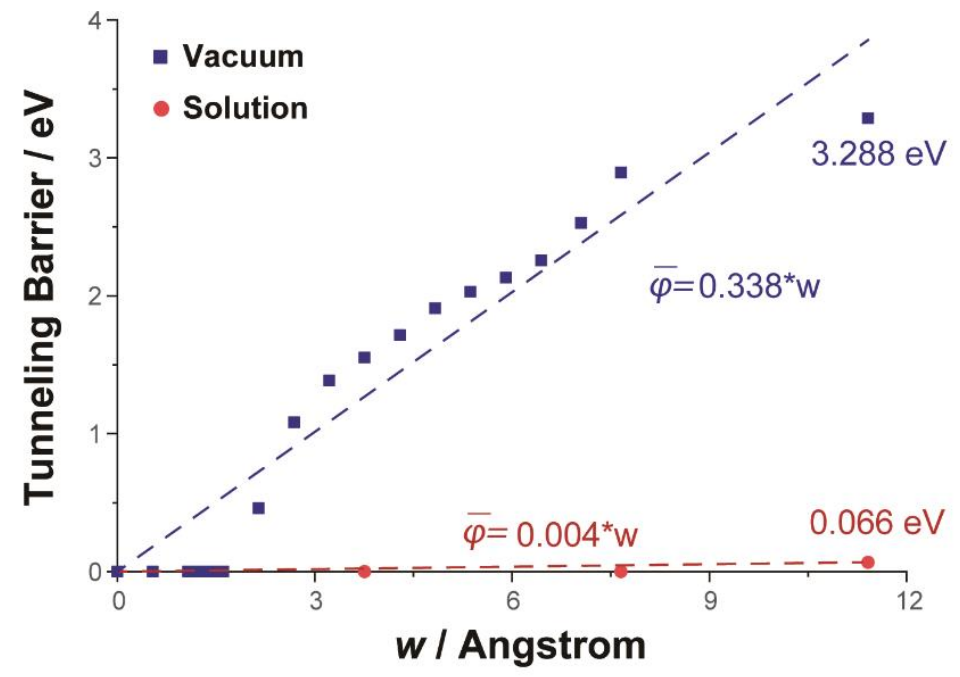

Figure S2. Calculated effective potential curve and the tunneling barrier energy. (a) the schematic of the effective potential between Ag NPs and Au UME using DFT calculation, while $\mathrm{Ag}, \mathrm{Au}, \mathrm{O}$ and $\mathrm{H}$ atoms are in blue, yellow, red and white balls, the effective potential curve in water solution and vacuum are illustrated by black solid and red dot lines, respectively. (b) the mean tunneling energy barriers at different interface distances (blue: vacuum condition, red: water condition).

In order to make the theoretical model more reasonable and practical, the barrier height in aqueous system is first estimated. The probability of electron tunneling in Simmons model $\left(P_{t}\right)$ could be achieved based on the well-known WBK approximation: ${ }^{1,2,13}$

$$
P_{t} \propto e^{-4 w \pi \sqrt{2 m \bar{\varphi}} / h}
$$

where $\bar{\varphi}$ is the mean tunneling barrier, $w$ is the thickness of the tunneling barrier, $m$ is the electronic mass, $h$ is the Planck constant. From the theoretical prospect of view, in the electron tunneling theory, $P_{t}$ follows the equation: 


$$
P_{t} \propto e^{-\beta w}
$$

where $\beta$ is a factor depending on the height of tunneling barrier and the nature of the medium between the two states, and it is also a range parameter that characterizes the distance dependence of the electron-transfer rate. Thus, for the electron tunneling between two metals, $\beta$ can be defined as: ${ }^{13}$

$$
\beta \approx 4 \pi(2 m \bar{\varphi})^{1 / 2} / h
$$

$\bar{\varphi}$ is the mean tunneling barrier, thus, $\bar{\varphi}$ could be obtained:

$$
\bar{\varphi} \approx \frac{\beta^{2} h^{2}}{32 \pi m}=0.95 \beta^{2}
$$

Generally, the values of $\beta$ measured at room temperature, typically range from 0.4 to $1 \AA^{-1}$ in aqueous system. ${ }^{14}$ Thus, the corresponding tunneling barrier is in the range of $0.15 \sim 1 \mathrm{eV}$. The tunneling barrier height in aqueous solvent of $0.15 \mathrm{eV}$ is used in theoretical simulation.

Based on eq. 37, we obtain that the decrease in the height of tunneling barrier contributes to the increase on the tunneling distance of electrons. In addition, previous publication has reported that the presence of water inside a capacitor junction can obviously lower the tunneling barrier, resulting in the unusually large tunneling distance. ${ }^{15}$ Herein, the medium between $\mathrm{Au}$ ultramicroelectrode and Ag Nanoparticles is electrolyte solution, which can greatly increase the tunneling distance of electrons compared with that in the vacuum environment.

To explore the effect of water on the tunneling barrier energy between a single Ag NP and $\mathrm{Au}$ electrode, we use DFT simulation to investigate the effective potential curves of the $\operatorname{Ag}(111)$ and the $\mathrm{Au}(111)$ surfaces in vacuum and water, respectively, aiming at providing a reasonable comparison of the tunneling energy barrier from the atomic level. First, the work functions of $\mathrm{Ag}(111)$ and $\mathrm{Au}(111)$ are calculated as 4.36 and $5.11 \mathrm{eV}$, respectively, which are consistent with the experimental results (4.26 eV for $\mathrm{Ag}(111)$ and $5.10 \mathrm{eV}$ for $\mathrm{Au}(111))$. Second, from the effective potential curves between the $\operatorname{Ag}(111)$ and $\mathrm{Au}(111)$ interface in water or vacuum condition calculated in Figure S2a, we can see that in the absence of water, the effective potentials between the interface at a distance $(w)$ about $11.5 \AA$ are rapidly rise to vacuum level (red dot line) and the maximum tunneling barrier height is nearly $4.36 \mathrm{eV}$. While, the effective potential becomes much lower and the oscillation of effective potential appears with the presence of 3 layers of water solution (black solid line). The maximum tunneling barrier height is only $0.10 \mathrm{eV}$. Finally, $\bar{\varphi}$ is the mean tunneling barrier height above the Fermi level energy, which could be calculated by the following equation. ${ }^{1}$ 


$$
\bar{\varphi}=\frac{1}{\Delta w} \int \varphi(w) d w
$$

The tunneling barriers are calculated to be 3.288 and $0.066 \mathrm{eV}$ for the vacuum and water solution, respectively. Moreover, we also consider the mean tunneling energy barrier at different interface distances $(w)$ as shown in Figure $\mathbf{S} 2 \mathrm{~b}$, one can see that, the mean tunneling barrier is as high as $1.385 \mathrm{eV}$ at typical tunneling distance $(\sim 3 \AA)$ in the absence of water solution, while there is even no addition tunneling with the presence of water. It indicates that the presence of water greatly reduces the mean tunneling energy barrier. Consequently, a much longer tunneling distance could be expected in water. 


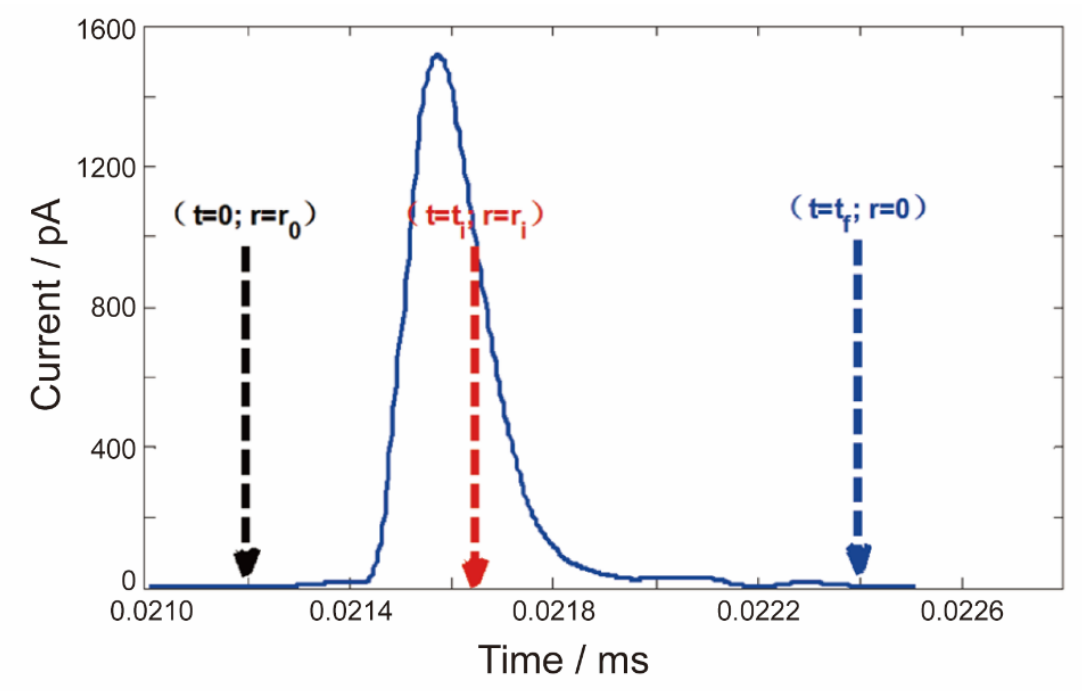

Figure S3. The time-resolved $i$-t trace in electro-oxidation of a single $20 \mathrm{~nm} \mathrm{Ag} \mathrm{NP.}$

The details of simulation. The electro-oxidation of single Ag NPs starts from beginning $\left(t_{0}\right)$ to the end $\left(t_{f}\right)$, the radius of Ag NPs $(r)$ changes from $r_{0}$ to 0 . The whole reaction is tracked by the time-resolved current trace, the charge $(Q)$ passed under the current spike can be calculated through the following equation that is derived from Faraday's First law:

$$
Q=\frac{4 \pi r_{0}^{3} F \rho}{3 M_{A g}}
$$

Also, the charge $(Q)$ can be obtained by integrating each experimental current spike:

$$
\mathrm{Q}=\int_{0}^{t_{f}} i(t) d t
$$

Combining eq. 41 and eq. 42, one can achieve:

$$
r_{0}=\sqrt[3]{\frac{3 M_{A g} \int_{0}^{t_{f}} i(t) d t}{4 \pi F \rho}}
$$

Similarly, for the reaction from any time $\left(t_{i}\right)$ to the end $\left(t_{f}\right)$, the radius of Ag nanoparticles $(r)$ ranges from $r_{i}$ to 0 . The radius of $\mathrm{Ag}$ nanoparticle can be deduced as expressed below:

$$
r_{i}=\sqrt[3]{\frac{3 M_{A g} \int_{t_{i}}^{t_{f}} i(t) d t}{4 \pi F \rho}}
$$

At any time, $t=t_{0}$, the radius of Ag nanoparticle $\left(r_{i}\right)$, the Faradaic current of the electrodissolution of an Ag NP follows: 


$$
i(t)=\frac{\partial Q(t)}{\partial t}=-\frac{F \rho}{M_{A g}} \frac{\partial V(t)}{\partial t}=-\frac{4 \pi r_{i}^{2} F \rho}{M_{A g}} \frac{\partial r_{i}}{\partial t}
$$

First, a simple oxidation model $\left(\partial r_{i} / \partial t\right)$ is adopted to illustrate the details of simulation. For example, the variation of rate in Ag NP size is described by a zero-order rate law with exponential dependence on the tunneling distance $\left(w_{i}\right)$ :

$$
\frac{\partial r_{i}}{\partial t}=-v_{r} e^{-\beta w_{i}}
$$

where $v_{r}$ is the electrochemical reaction rate, $\beta^{-1}$ is the tunneling constant. Combining eq. 45 and eq. 46 yields:

$$
W_{i}=-\frac{\ln \frac{i(t) M_{A g}}{4 \pi r_{i}^{2} F \rho v_{r}}}{\beta}
$$

Therefore, based on the time-resolved ( $i$-t) curves of single Ag NPs collision, $r_{i}$ at any time can be obtained by eq. 44 . Also, $w_{i}$ at any time can be obtained by eq. 47 .

For Figure 2a, the electro-oxidation of single Ag NPs is more complicated as shown in eq. 7 and $e q .8$ in the main text, which contains the unknown $U_{p}$ related to the $w_{i}$. Therefore, it is necessary to further combine the steady state condition $\left(i_{t u n}=i_{o x}\right)$, and solve the position and the potential of Ag NPs by numerical solution. In this case, the change of the distance leads to the change of the experimental current. Therefore, the value of distance can be deduced by the experiment current value. For Figure $2 \mathrm{~b}$ and $\mathrm{c}, w_{i}$ and $\mathrm{r}_{\mathrm{i}}$ are first assigned. $U_{p}$ is determined by combining eq. 5 in the main text with eq. 14 in Supplementary Note $2\left(i_{t u n}=i_{o x}\right)$. 

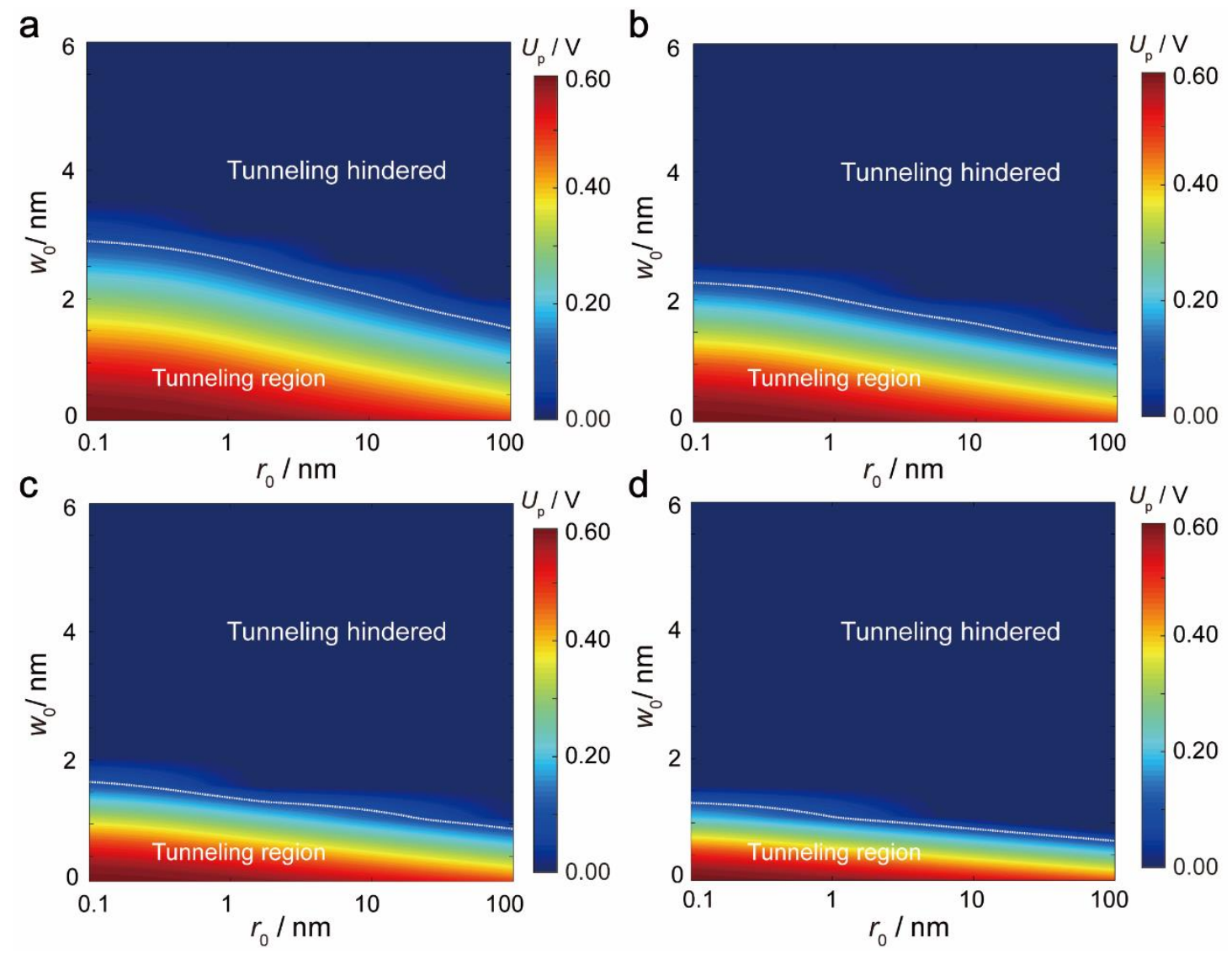

Figure S4. Simulated 2D contour map of the dynamic potential distribution at the UME interface in the process of single Ag NPs stochastic collision electrochemistry. The tunneling barrier $\bar{\varphi}$ is set as (a) $0.1 \mathrm{eV}$, (b) $0.2 \mathrm{eV}$, (c) $0.5 \mathrm{eV}$, (c) $1.0 \mathrm{eV}$. Numerical simulation according to $j_{\text {tun }}=j_{o x}$ as a function of $w_{0} / r_{0}, U_{e l}=+600 \mathrm{mV}$ vs Ag/AgCl QRCE and $v_{r}=5 \times 10^{4}$ $\mathrm{nm} \mathrm{s}^{-1}$.

The different barrier heights are also used in theoretical simulation. According to eq. 39, the tunneling barrier height ranges from $0.15 \mathrm{eV}$ to $1 \mathrm{eV}$ in aqueous system. Thus, four values as typical tunneling barrier heights, consisting of $0.1 \mathrm{eV}, 0.2 \mathrm{eV}, 0.5 \mathrm{eV}$, and $1.0 \mathrm{eV}$ are considered here for the theoretical simulation. The simulated results are displayed in Figure S4, all 2D contour maps indicate that $U_{p}$ for four different tunneling barrier heights exhibit same variation trend as the dynamic shrinkage of Ag NPs. 


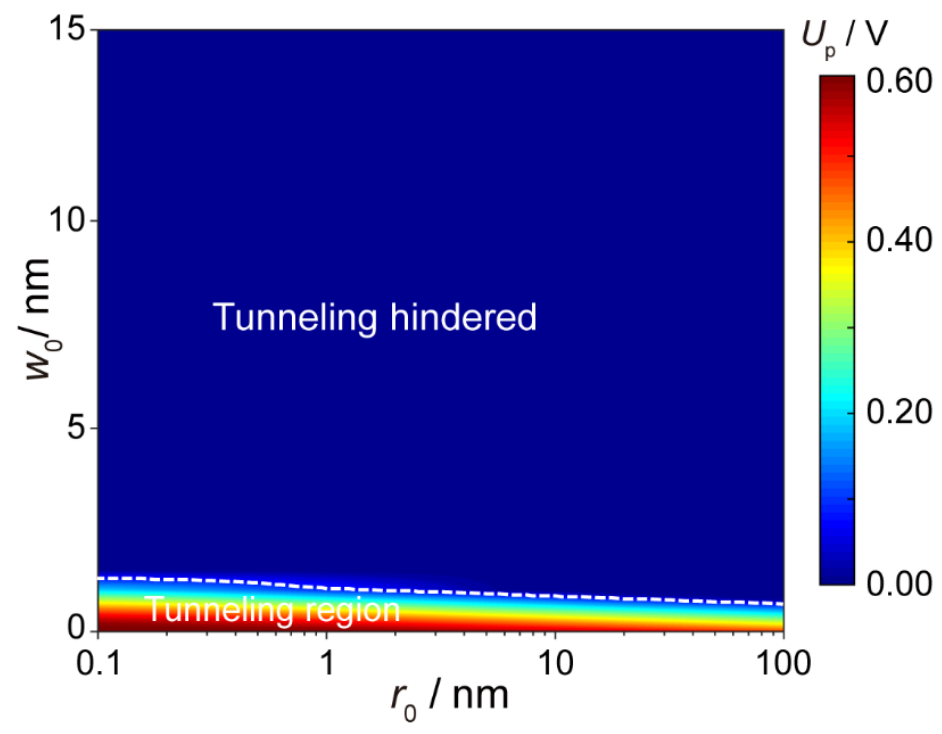

Figure S5. Potential distribution at the electrode interface in the $\mathrm{Ag}-\mathrm{TiO}_{2}$ film-Au. Numerical simulation according to $j_{t u n}=j_{o x}$ as a function of $w_{0} / r_{0}$ for $\bar{\varphi}=1 \mathrm{eV}, U_{e l}=+600 \mathrm{mV} v s$ $\mathrm{Ag} / \mathrm{AgCl} \mathrm{QRCE}$ and $v_{r}=5 \times 10^{4} \mathrm{~nm} \mathrm{~s}^{-1} .^{3}$ 

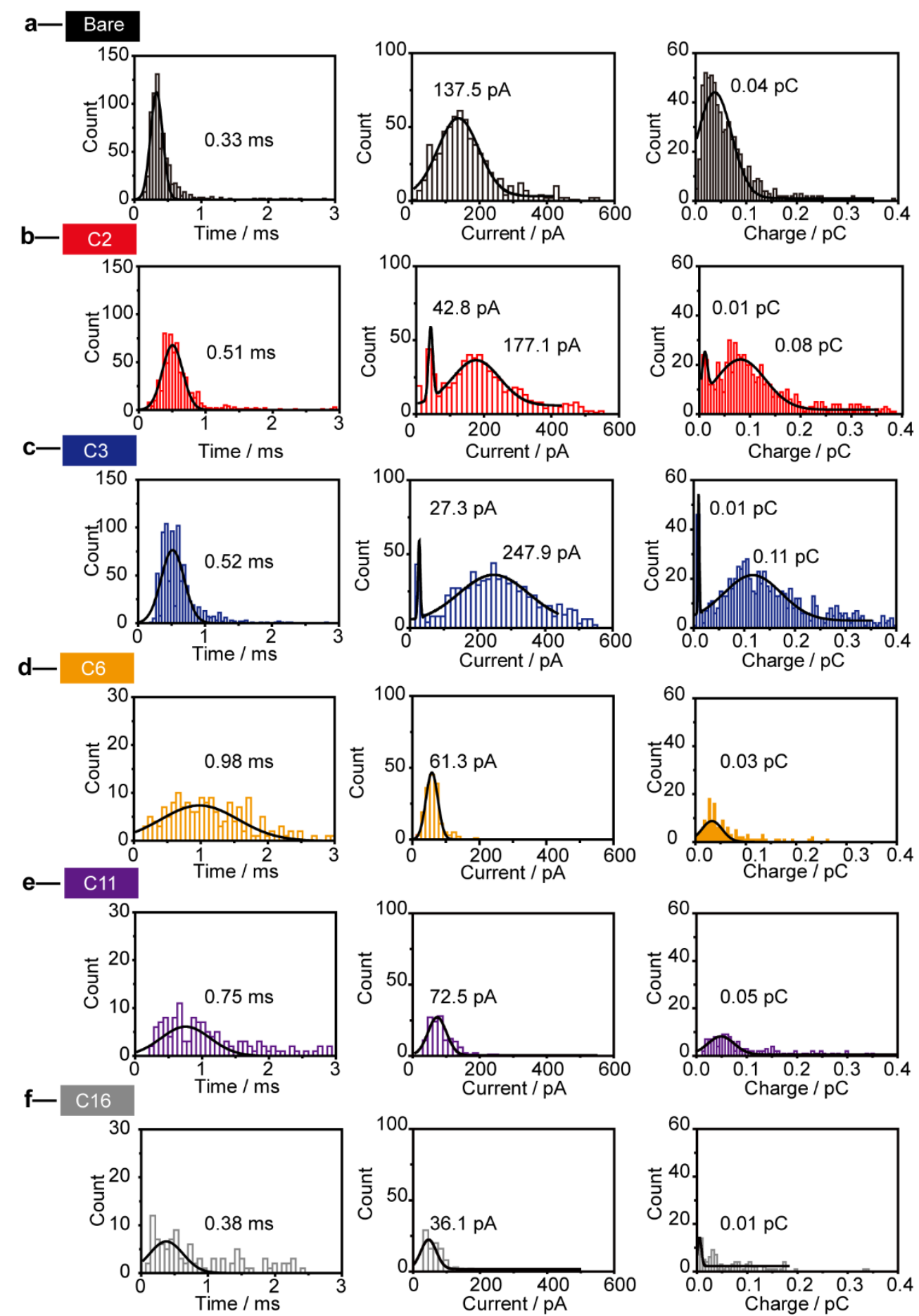

Figure S6. Histograms of the durations, peak currents and charges of stochastic collision electrochemistry of $10 \mathrm{~nm}$ radius Ag NPs with different SAMs modification. Histograms showing the distributions of the durations, peak currents and charges of $10 \mathrm{~nm}$ radius Ag NPs with different $\mathrm{CH}_{2} \mathrm{SH}\left(\mathrm{CH}_{2}\right)_{n-1} \mathrm{COOH}$ SAMs modification of (a) bare, (b) $n=1$, (c) $n=2$, (d) $n$ $=5$, (e) $n=10$, (f) $n=15$. Black curves show Gaussian fits. The data were obtained from the 
chronoamperometry curves from a large population of oxidation events of individual Ag NPs (more than 1000 events). Detailed molecular formula of SAMs is listed in Table S1.

The charge passed under the current spike can be calculated through the following equation that is derived from Faraday's First law:

$$
D_{N P}=2 \sqrt[3]{\frac{3 Q M_{A g}}{4 \pi F \rho}}
$$

where $D_{N P}$ is the diameter of a $\mathrm{Ag} \mathrm{NP}, Q$ is the integrated charge of a single current spike, $M_{A g}$ is the atomic molecular mass of $\mathrm{Ag}$ of $107.9 \mathrm{~g} \mathrm{~mol}^{-1}, F$ is the Faraday constant of 96485 $\mathrm{C} \mathrm{mol}^{-1}$, and $\rho$ is the density of silver of $10.5 \times 10^{6} \mathrm{~g} \mathrm{~m}^{-3}$. The charge resulted from the electrooxidation of $20 \mathrm{~nm} \mathrm{Ag} \mathrm{NPs} \mathrm{is} \mathrm{estimated} \mathrm{to} \mathrm{be} 0.04 \mathrm{pC}$ according to eq. 48 , which is quite close to the statistic value shown in Figure S5a. Also, note that the charge, frequency and the amplitude of predominate events owing to the stochastic collision of Ag NPs obviously decreases with the increase of the alkyl chain length (Figure 3a and Figure S5).

Simmons model used here describes the electric tunneling effect between electrodes separated by a thin insulating film. Also, it defines the tunneling current-voltage characteristic of the electrode junctions. ${ }^{1,2}$ The probability of electron tunneling in Simmons model $P_{t}$ could be achieved based on the well-known WBK approximation: ${ }^{1,2,13}$

$$
P_{t} \propto e^{-4 w \pi \sqrt{2 m \bar{\varphi}} / h}
$$

where $\bar{\varphi}$ is mean tunneling barrier, $w$ is thickness of the tunneling barrier, $m$ is electronic mass, $h$ is Planck constant.

The extended electron transfer theory discusses the electron transfer at different distance from redox moiety and metal electrode, where the electron transfer is usually deemed as tunneling of electron between states on the redox moiety and those in the electrode. ${ }^{13}$ This theory also describes the relationship between the electron transfer-rate constant and distance as well as the nature of the intervening medium. ${ }^{14}$ Based on the experiment research and measurement, the appropriate expression of the possibility of electron tunneling $\left(P_{t}\right)$ is reasonable to be suggested as:

$$
P_{t} \propto e^{-\beta w}
$$

where $\beta$ is a factor depending on height of tunneling barrier and the nature of the medium between the states, and the values of $\beta$ are of the order of $1 \AA^{-1}$. It depends primarily upon the 
nature of the intervening medium. According to previous study, for the electron tunneling between two pieces of metal through vacuum environment, $\beta$ could be defined as: ${ }^{13}$

$$
\beta \approx 4 \pi(2 m \bar{\varphi})^{1 / 2} / h
$$

which could be achieved by combining eq. 49 and eq. 50. In this case, $\bar{\varphi}$ could be estimated by the work function of the metal. Such as, for $\mathrm{Ag}, \bar{\varphi}$ is $4.26 \mathrm{eV}, \beta$ is about $2.11 \AA^{-1}$.

Therefore, we could believe both the Simmons model and the extended electron transfer theory give insight into the tunneling of electron between two states, and the increase in the distance from Ag NPs to Au UME could decrease the possibility of tunneling. Here, there are two contributions to the distance, $\mathrm{d}=\mathrm{d} 1+\mathrm{d} 2$, where $\mathrm{d} 1$ is the SAMs length and $\mathrm{d} 2$ is the distance from the end of SAMs to Au UME surface, respectively. The longer the carbon chain length, the lower the possibility of electron tunneling, thus, the decrease in the potential at the UME interface (potential applied at a Ag NP). Consequently, the low potential results in the occurrence of incomplete oxidation of Ag NPs, contributing to the decrease in the integrated charge, amplitude and frequency of spike with increasing the chain length.

It is worth noting that the duration time of transient current is also governed by the chain length of SAMs. The duration first undergoes increase with increasing the chain length and then it decreases when the number of carbon increases to six. We assign this variation in duration to the following reasons. The interaction between Ag NPs and Au UME typically become strong due to the SAM coating, thus elongating the dwell time of the particles on the surface of $\mathrm{Au}$ UME. ${ }^{12}$ For longer chain length of $\mathrm{C} 11$ and $\mathrm{C} 16$, it is difficult for electrons to across the tunneling energy barrier, suppressing the electron transfer. Consequently, it lowers the potential applied at a Ag NP, and the rate of electro-oxidation deceases, thus, the decrease in durations of events. ${ }^{16}$ 


\section{References}

1. Simmons, J. G. J. Appl. Phys. 1963, 34, 2581-2590.

2. Simmons, J. G. J. Appl. Phys. 1963, 34, 1793-1803.

3. Hill, C. M.; Kim, J.; Bard, A. J. J. Am. Chem. Soc. 2015, 11321-11326.

4. Perdew, J. P.; Burke, K.; Ernzerhof, M. Phys. Rev. Lett. 1996, 77, 3865-3868.

5. Kwon, S. J.; Bard, A. J. J. Am. Chem. Soc. 2021, 134, 7102-7108.

6. Thonhauser, T. et al. Phys. Rev. B. 2007, 76, 125112.

7. Klimeš, J.; Bowler, D. R.; Michaelides, A. J. Phys. Condens. Matter. 2010, 22, 074203.

8. Kihm, K. D.; Banerjee, A.; Choi, C. K.; Takagi, T. Exp. Fluids. 2004, 37, 811-824.

9. Kresse, G.; Joubert, D. F. Phys. Rev. B. 1999, 59, 1758-1775.

10. Tomasi, J.; Mennucci, B.; Cammi, R. Chem. Rev. 2005, 105, 2999-3094.

11. Gray, C. M.; Saravanan, K.; Wang, G.; Keith, J. A. Mol. Simul. 2017, 43, 420-427.

12. Ma, H.; J.-F. Chen.; H.-F. Wang.; P.-J. Hu.; W. Ma.; Y.-T. Long. Nat. Commun. 2020, 11, 2307.

13. Bard, A. J.; Faulkner, L. R. Electrochemical Methods: Fundamentals and Applications, $2^{\text {nd }}$ ed.; Wiley: New York, 2001, p115-136.

14. Gavaghan, D. J.; Feldberg, S. W. Extended electron transfer and the Frumkin correction. J. Electroanal. Chem. 2000, 491, 103-110.

15. Hahn, J. G.; Hong, Y. A.; Kang, H. Appl. Phys. A 1998, 66, 467-472.

16. Xiao, X. Y.; Pan, S.; Jang, J. S.; Fan F-R F.; Bard, A. J. Single Nanoparticle Electrocatalysis: Effect of Monolayers on Particle and Electrode on Electron Transfer. J. Phys. Chem. C 2009, 113, 14978-14982. 